\title{
Stereotyped Combination of Hearing and Wind/Gravity-Sensing Neurons in the Johnston's Organ of Drosophila
}

\author{
Yuki Ishikawa, Mao Fujiwara, Junlin Wong, Akari Ura and Azusa Kamikouchi* \\ Division of Biological Science, Graduate School of Science, Nagoya University, Nagoya, Japan
}

The antennal ear of the fruit fly, called the Johnston's organ $(\mathrm{JO})$, detects a wide variety of mechanosensory stimuli, including sound, wind, and gravity. Like many sensory cells in insect, JO neurons are compartmentalized in a sensory unit (i.e., scolopidium). To understand how different subgroups of $\mathrm{JO}$ neurons are organized in each scolopidial compartment, we visualized individual $\mathrm{JO}$ neurons by labeling various subgroups of JO neurons in different combinations. We found that vibration-sensitive (or deflection-sensitive)

OPEN ACCESS

Edited by: Paivi H. Torkkeli,

Dalhousie University, Canada

Reviewed by: Daniel F. Eberl,

The University of lowa, United States Joerg T. Albert,

University College London, United Kingdom

*Correspondence: Azusa Kamikouchi kamikouchi@bio.nagoya-u.ac.jp

Specialty section:

This article was submitted to Invertebrate Physiology, a section of the journa Frontiers in Physiology

Received: 09 October 2019 Accepted: 09 December 2019

Published: 08 January 2020

Citation:

Ishikawa Y, Fujiwara M, Wong J, Ura A and Kamikouchi A (2020) Stereotyped Combination of Hearing and Wind/Gravity-Sensing Neurons in the Johnston's Organ of Drosophila.

Front. Physiol. 10:1552.

doi: 10.3389/fphys.2019.01552 neurons rarely grouped together in a single scolopidial compartment. This finding suggests that $\mathrm{JO}$ neurons are grouped in stereotypical combinations each with a distinct response property in a scolopidium.

Keywords: ear, mechanosensory neuron, fruit fly, scolopidium, compartment

\section{INTRODUCTION}

The ability to sense our surrounding environment is crucial for survival. Animals have developed specialized sensory organs to detect various external and internal signals. The ear is an example of a specialized organ that detects sound; in mammals, the ears are also responsible for balance. Some insect species also have a specialized hearing organ, the insect ear, which is located on different body parts, including the head, wings, thorax, abdomen, and legs (Gopfert and Hennig, 2016). Studies of the insect hearing system have yielded important findings ranging from the biophysics of sound perception to auditory signal processing in the brain (Coen and Murthy, 2016; Gopfert and Hennig, 2016).

The fruit fly Drosophila melanogaster is a useful model for studying the cellular and circuit mechanisms of hearing and other types of mechanosensory processing (Patella and Wilson, 2018). In fruit flies, the ear is comprised of the antennal receiver and hearing organ called the Johnston's organ (JO) (Figure 1A). The JO, which is located at the second antennal segment, is the largest mechanosensory organ in fruit flies. Stimuli from an external source, such as sound, wind, or gravity, induce the movement of the antennal receiver, which activates the mechanosensory neurons in the JO, the JO neurons. JO neurons serve a vital role in the fruit fly behavior, such as the locomotor change in response to courtship sound, wind-induced suppression of locomotion, anti-geotaxis behavior, and flight control (Kamikouchi et al., 2009; Yorozu et al., 2009; Mamiya and Dickinson, 2015).

The fruit fly's JO neurons are classified into five subgroups, JO-A to JO-E, according to their distinct projection patterns in the brain (Kamikouchi et al., 2006). These subgroups can further 

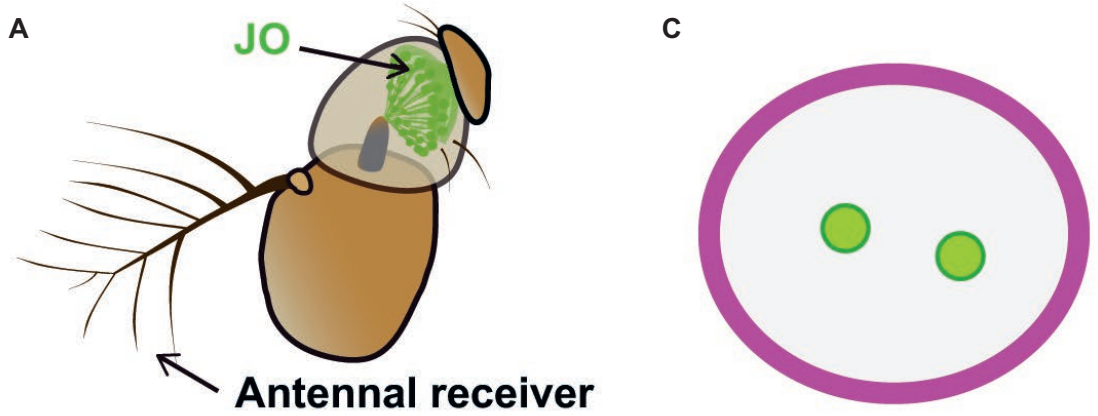

B

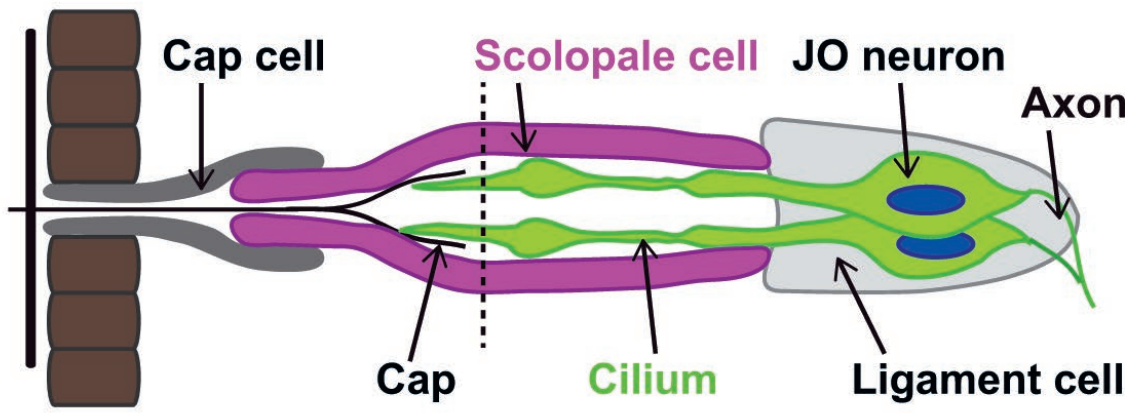

D
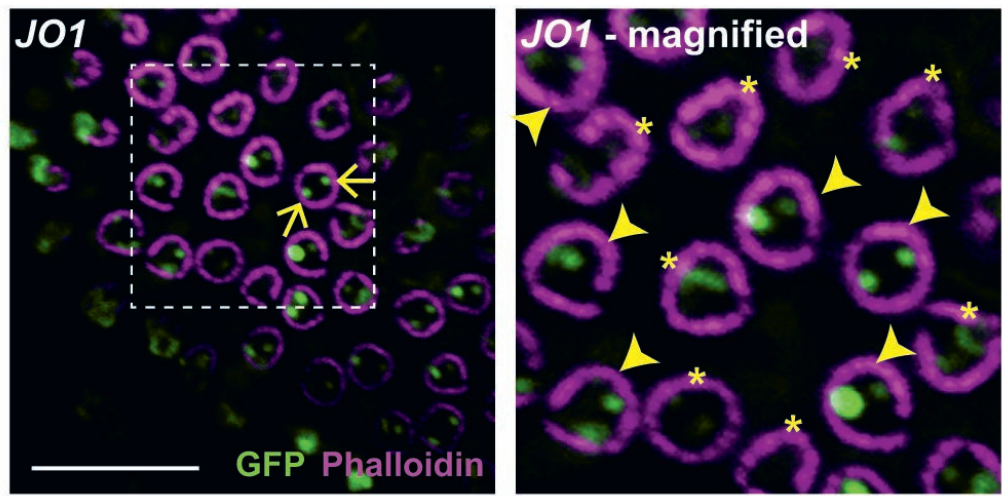

E
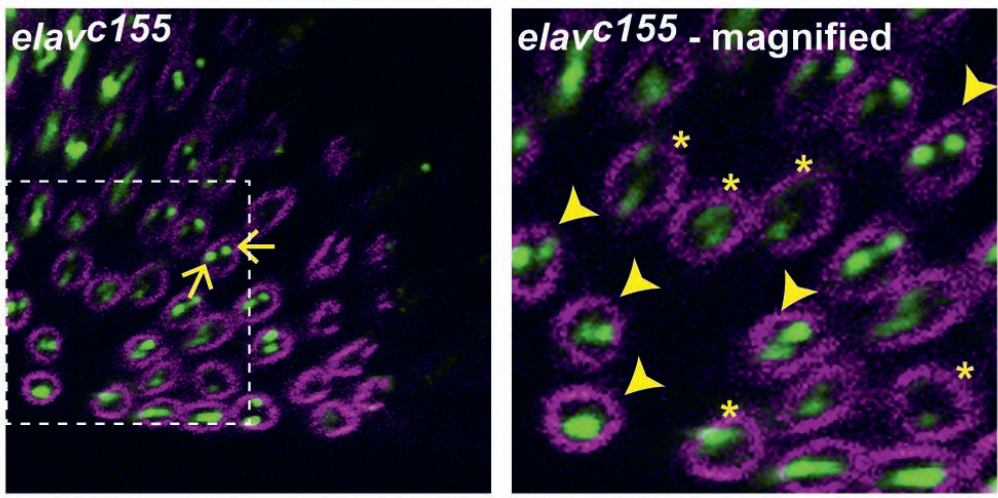

FIGURE 1 | Labeled JO neurons in a scolopidium. (A) The antennal ear of fruit flies. Johnston's organ (JO) is housed in the second antennal segment.

(B) Scolopidium in the JO. A schema of a section at the dashed horizontal line is shown in (C). (C) Horizontal view of a scolopidium. Labeled cilia are visualized as dots (green) in a scolopidium (magenta). (D,E) Labeled JO neurons in GAL4 driver strains that label all JO subgroup neurons. Scolopidia containing JO1- (D) and elav ${ }^{155}$-labeled cilia (E). Arrows indicate the examples of labeled cilia (green) in a scolopidium (magenta) (left panels). Dotted square indicates the area that is magnified in the right panel. Arrowheads and asterisks in the magnified views indicate the selected and not-selected scolopidia, respectively (right panels). Scale bar $=10 \mu \mathrm{m}$. Panels (A) and (B) were modified from Ishikawa and Kamikouchi (2016) and Matsuo et al. (2016) with permissions. 
be categorized into vibration-sensitive and deflection-sensitive neurons (Kamikouchi et al., 2009; Yorozu et al., 2009). JO-A and JO-B neurons are vibration-sensitive, and function as sound sensors. Their response is frequency-dependent, ranging from $\sim 10 \mathrm{~Hz}$ up to $\sim 1,000 \mathrm{~Hz}$ when measured together. When measured separately, the JO-B neurons show a low frequency preference at about $<100 \mathrm{~Hz}$, while JO-A neurons preferentially respond to higher frequency (Matsuo et al., 2014; Patella and Wilson, 2018). On the other hand, JO-C and JO-E are activated maximally by static deflection of the antennal receiver, and serve as the major gravity and wind sensors. The precise function of JO-D neurons has not yet been determined due to their small total percentage ( $<30$ neurons) and the lack of specific driver strains. Both vibration and deflection stimuli activate JO-D neurons (Matsuo et al., 2014).

An interesting feature of JO neurons is their compartmentalization; that is, two or three JO neurons are grouped together in specialized structures known as scolopidia (Todi et al., 2004). Each JO scolopidium acts as a sensory unit that helps to connect the apical sensory cilium to the joint cuticle. The scolopale cell wraps around the cilia of JO neurons and provides a sealed environment for maintaining and regulating the ionic composition of the endolymph (Figure 1B; Boekhoff-Falk and Eberl, 2014). Although previous findings suggest that JO neurons belonging to the same scolopidium should belong to heterogeneous subgroups (Kamikouchi et al., 2006), it remains unclear how different subgroups of JO neurons are organized in each scolopidium.

Similar examples of compartmentalized sensory neurons can be found in the sensilla that house the olfactory receptor neurons (ORNs) of Drosophila, the $\mathrm{CO}_{2}$-senstitive ORN of the malaria mosquito Anopheles, and the taste sensilla of flies and other insects, where the primary sensory neurons are grouped in stereotyped combinations (de Bruyne et al., 2001; Lu et al., 2007; Freeman and Dahanukar, 2015). In insect olfactory sensilla, it is predicted that short-term activation of one ORN may interfere with the signaling of a neighboring ORN (Nikonov and Leal, 2002; Vermeulen and Rospars, 2004), and in the case of Drosophila, transient excitation of one ORN inhibits the prolonged activation of the neighboring ORN (Su et al., 2012). This interaction between compartmentalized ORNs does not require a synaptic interaction, and might reflect ephaptic coupling.

To investigate how the different subgroups of JO neurons are organized in each scolopidium, we examined the distribution of JO neurons housed within the scolopidia. Our findings suggest that vibration- and deflection-sensitive JO neurons are housed as a pair in each scolopidium of the JO.

\section{MATERIALS AND METHODS}

\section{Fly Stocks and Culture}

Flies were reared on yeast/cornmeal/agar medium at $25^{\circ} \mathrm{C}$. The GAL4 strains that label JO neurons (Kamikouchi et al., 2006; Ishikawa et al., 2017) are shown in Table 1. elav ${ }^{C 155}$ was used as a pan-neuronal GAL4 driver (RRID: BDSC_458, obtained from the Bloomington Drosophila Stock Center). UAS-nompCL-GFP8 (Cheng et al., 2010), a gift from Dr. Y. N. Jan, was used as a reporter strain to express NompC-L-GFP.

\section{Immunohistochemistry}

Female flies at 7-14 days after eclosion were used for dissection. Immunolabeling was performed as described previously with minor modifications (Matsuo et al., 2014). In brief, antennae were fixed with $4 \%$ paraformaldehyde in phosphate-buffered saline for 60-90 $\mathrm{min}$ on ice and labeled with antibody. As primary antibodies, we used rabbit green fluorescent protein (GFP) polyclonal antibody (Invitrogen, La Jolla, CA, \#A11122; RRID: AB_221569; 1:1,000 dilution) to detect NompC-L-GFP and rat Elav antibody (Hybridoma Bank, Rat-Elav-7E8A10 anti-elav; 1:250 dilution) to detect JO neuron cell bodies. As secondary antibodies, we used Alexa Fluor 488-conjugated

TABLE 1 | Labeling patterns of GAL4 driver strains and the number of labeled JO neurons in each scolopidium.

\begin{tabular}{|c|c|c|c|c|c|c|c|c|}
\hline \multirow[t]{2}{*}{ Group } & \multirow[t]{2}{*}{ GAL4 strains } & \multirow{2}{*}{$\begin{array}{l}\text { Labeled } \\
\text { subgroups }\end{array}$} & \multirow{2}{*}{$\begin{array}{l}\text { Observed } \\
\text { antennas }\end{array}$} & \multirow{2}{*}{$\begin{array}{l}\text { Countable } \\
\text { scolopidia }\end{array}$} & \multirow{2}{*}{$\begin{array}{c}\text { Selected } \\
\text { scolopidia }\end{array}$} & \multicolumn{3}{|c|}{ Number of selected scolopidia } \\
\hline & & & & & & $1 \mathrm{JO}$ neuron & $2 \mathrm{JO}$ neurons & $3 \mathrm{JO}$ neurons \\
\hline & JO1 & $A, B, C, D, E$ & 3 & 99 & 65 & $1(1.5 \%)$ & $64(98.5 \%)$ & $0(0 \%)$ \\
\hline & elav ${ }^{C 155}$ & $\mathrm{~A}, \mathrm{~B}, \mathrm{C}, \mathrm{D}, \mathrm{E}$ & 11 & 123 & 69 & $3(4.35 \%)$ & $65(94.2 \%)$ & $1(1.45 \%)$ \\
\hline \multirow[t]{4}{*}{1} & $J 015$ & A, B (most) & 4 & 161 & 77 & $75(97.4 \%)$ & $2(2.6 \%)$ & $0(0 \%)$ \\
\hline & J031 & C, E (most) & 3 & 117 & 54 & $54(100 \%)$ & $0(0 \%)$ & $0(0 \%)$ \\
\hline & JO32 & C, E (most) & 3 & 176 & 90 & $87(96.7 \%)$ & $3(3.3 \%)$ & $0(0 \%)$ \\
\hline & $J 015+J 031$ & $A, B, C, E$ & 8 & 229 & 88 & 64 (72.7\%) & $21(23.9 \%)$ & $3(3.4 \%)$ \\
\hline \multirow[t]{6}{*}{2} & $R 74 C 10$ & $\mathrm{~A}$ (most) & 26 & 468 & 108 & $91(84.3 \%)$ & $16(14.8 \%)$ & $1(0.9 \%)$ \\
\hline & $\mathrm{JO22}$ & A (a few) & 3 & 117 & 4 & $4(100 \%)$ & $0(0 \%)$ & $0(0 \%)$ \\
\hline & JO24 & A (a few) & 4 & 226 & 28 & $28(100 \%)$ & $0(0 \%)$ & $0(0 \%)$ \\
\hline & JO26 & A (a few) & 5 & 278 & 47 & $47(100 \%)$ & $0(0 \%)$ & $0(0 \%)$ \\
\hline & $\mathrm{JO2}$ & B (most) & 7 & 206 & 77 & 77 (100\%) & $0(0 \%)$ & $0(0 \%)$ \\
\hline & $R 74 C 10+J O 2$ & $A, B$ & 5 & 290 & 107 & $101(94.4 \%)$ & $6(5.6 \%)$ & $0(0 \%)$ \\
\hline
\end{tabular}

Group 1 and group 2 are GAL4 driver strains that label multiple and single subgroups of JO neurons, respectively. Group-1 GAL4 driver strains label most JO-A and JO-B (JO15) or JO-C and JO-E (JO31 and JO32) (Kamikouchi et al., 2006). In group 2, R74C10 and JO2 label most JO-A and JO-B neurons, respectively (Kamikouchi et al., 2006; Ishikawa et al., 2017). JO22, JO24, and JO26 label a few JO-A neurons (Ishikawa et al., 2017). 
anti-rat IgG (Jackson ImmunoResearch, Cambridgeshire, UK, \#112-545-167; RRID: AB_2338362; 1:300 dilution) and Alexa Fluor 647-conjugated anti-rabbit IgG (Thermo Fisher Scientific, \#A21245; RRID: AB_2535813; 1:300 dilution). Alexa Fluor 555 phalloidin (Thermo Fisher Scientific, \# A34055; 1:50 dilution) was used to visualize F-actin.

\section{Visualization of Antennal Morphology}

For observation of the scolopidia from various angles, each of the second antennal segments was mounted on a slide glass at a different orientation except for the ventral and backward sides up; observation from these sides gives obscure images and thus we avoided these angles. Each sample was viewed under a confocal laser scanning microscope (FLUOVIEW FV1000D, Olympus, Japan) equipped with a silicone-oil immersion 60x Plan-Apochromat objective lens $(\mathrm{NA}=1.30$, UPLSAPO $60 \mathrm{xS}$, Olympus). Each sample was scanned twice; the first series was to observe the overall structure and to locate the scolopidia and JO neurons with a resolution of $512 \times 512$ pixels $(0.295 \mu \mathrm{m} /$ pixel $)$, and the second series was to focus on specific clusters of scolopidia and JO neurons with a resolution of $640 \times 640$ pixels $(0.110 \mu \mathrm{m} /$ pixel $)$.

\section{Quantification of the Number of Labeled Johnston's Organ Neurons in Scolopidia}

The number of labeled JO neurons in each scolopidium was quantified by analyzing the confocal data series. Because the scolopidia are arranged in a radial fashion in the second antennal segment, each individual scolopidium is oriented at a different angle in a single image (Kamikouchi et al., 2006). The scolopidia that are mounted perpendicular to the cover glass appear as a circle while the others appear as ovals or rods when observed in the confocal sections. The cilia of JO neurons within the scolopidia with a circular appearance are visualized as dots when labeled and can be accurately quantified by counting the number of dots in each circle (Figure 1C). In contrast, quantification of the number of labeled JO neurons in the scolopidia appearing as a rod shape is unreliable due to the overlapping of multiple JO neurons and scolopidia in the same spot. To accurately count the number of labeled JO neurons in each scolopidium, only the scolopidia mounted perpendicular to the cover glass, thus appearing as a circle, were quantified in this study. At a certain confocal section, we sometimes observed only one labeled JO neuron, even the scolopidium actually housed two labeled JO neurons, which could be observed at a different section. This could be due to termination of the cilia of two JO neurons in each scolopidium at different levels along the longitudinal axis of the scolopidium (Uga and Kuwabara, 1965). To avoid underestimating the number of labeled JO neurons in each scolopidium due to the different cilia lengths, we scanned the scolopidium along its longitudinal axis so that cilia of all the labeled JO neurons could be observed.

Statistical analyses were performed using $\mathrm{R}$ (version 3.3.3). The ratio of single/double/triple-labeled neuron populations was compared by Fisher's exact test. Values of $p$ were adjusted using the Bonferroni method for multiple comparisons.

\section{RESULTS}

\section{Visualization of Labeled Cilia in Johnston's Organ Scolopidia}

In the JO of Drosophila, $\sim 10 \%$ of the scolopidia contain three JO neurons scattered throughout the JO, whereas the remaining scolopidia contain two JO neurons (Todi et al., 2004). To clarify how different subgroups of JO neurons are distributed within each scolopidium, we visualized the cilia of specific JO neurons using the GAL4/UAS system to express NompC-L-GFP reporters (Cheng et al., 2010). First, to evaluate whether the NompCL-GFP marker dependably visualizes the cilia of labeled JO neurons, we used two GAL4 strains that label most JO neurons: JO1 (a.k.a. NP0761), which labels 94\% of JO neurons (Kamikouchi et al., 2006), and $e l a v^{C 155}$, which is widely used as a pan-neuronal GAL4 driver.

To count the number of labeled cilia in each scolopidium, we used the scolopidia that appeared perpendicular to the confocal scanning plane for the analysis (Figure 1D). At this angle, each scolopidium was visualized as a circle and the cilia of JO neurons within it appeared as dots, which were easy to quantify (see section "Materials and Methods" for details). First, we screened the countable scolopidia visualized as circles. Next, we selected scolopidia in which at least one labeled cilium was clearly visualized among the countable scolopidia to avoid underestimating the proportion of labeled JO neurons by including the inappropriate angles or poor antibody permeable samples. Finally, we counted the number of labeled cilia and obtained the percentages of one, two, and three labeled JO neurons among the selected scolopidia.

We evaluated $\sim 100$ countable scolopidia in each of two GAL4 strains. Approximately half of them exhibited strong and clear signals of one or more labeled cilia and were thus regarded as selected scolopidia, whereas the other half exhibited obscure signals, which were excluded from the following quantification (Figures 1D,E). More than $90 \%$ of the selected scolopidia had two labeled JO neurons in both strains (Figures 1D,E; Table 1). A few scolopidia (<5\%) had only one labeled JO neuron in both GAL4 strains and scolopidia that had three labeled JO neurons were observed only when elav ${ }^{C 155}$ was used. On the basis of these findings, we concluded that the NompC-L-GFP reporter system was useful for evaluating the ratio of one, two, and three labeled JO neurons in a single scolopidium, although not all scolopidia could be evaluated.

\section{Organization of Vibration-Sensitive and Deflection-Sensitive Johnston's Organ Neurons}

To reveal the combination of JO neuronal subgroups in each scolopidium, we used other GAL4 strains, each of 
which labels subsets of JO neuronal subgroups (Table 1; Kamikouchi et al., 2006; Ishikawa et al., 2017). We divided eight GAL4 strains into two groups according to the combination of labeled subgroups (Table 1). The first group, group 1, comprised strains that labeled multiple subgroups categorized as either vibration-sensitive or static deflection-sensitive JO neurons. The second group, group 2, comprised strains that labeled one single subgroup of vibration-sensitive JO neurons.

We first observed group-1 GAL4 strains to analyze the organization of vibration-sensitive (JO-A and JO-B) and deflection-sensitive (JO-C and JO-E) subgroups in each scolopidium (Figure 2A, Table 1). JO15, which labels most (but not all) JO-A and JO-B neurons (Kamikouchi et al.,
2006), labeled only one JO neuron in most selected scolopidia (97.4\%). Likewise, JO31 and JO32, both of which label most JO-C and JO-E (Kamikouchi et al., 2006), labeled only one JO neuron in most scolopidia (100\% in JO31 and $96.7 \%$ in JO32; Table 1). These results together suggest that the majority of scolopidia each contain one vibration and one static-deflection JO neuron as a pair.

To test this speculation, we combined two group-1 GAL4 strains, each of which labels either vibration-sensitive or deflection-sensitive JO neuronal subgroups. When JO15 and JO31 were combined, the ratio of single/double/triple-labeledneuron populations was significantly altered; single-labeled neuron populations decreased from 97.4 and 100 to $72.7 \%$ $(p=0.00002$ for JO15 vs. JO15 $+J 031, p=0.00001$ for $J O 31$
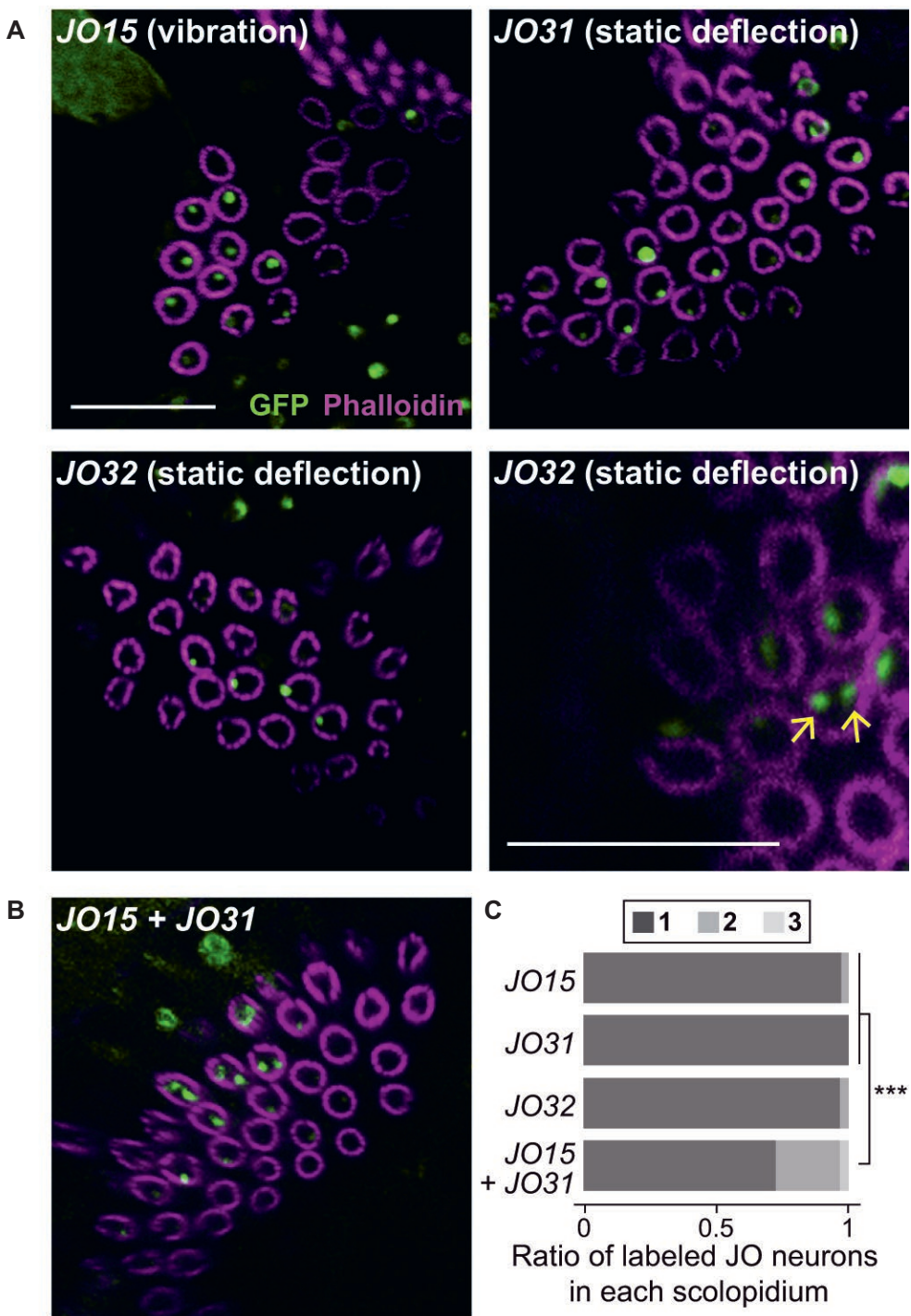

FIGURE 2 | Organization of vibration- and deflection-sensitive JO neurons in scolopidia. Labeled JO neurons in group-1 GAL4 driver strains that label vibration- or deflection-sensitive subgroups of $\mathrm{JO}$ neurons (A) and their combination (B). Arrows indicate the examples of two-labeled cilia (green) in a scolopidium (magenta) of JO32 strain (panel A, bottom-right panel). Scale bar $=10 \mu \mathrm{m}$. (C) The ratio of one, two, and three labeled JO neurons per scolopidium in each GAL4 driver strain.

${ }^{\star * *} p<0.001$, Fisher's exact test with the values of $p$ adjusted using the Bonferroni method for multiple comparisons. 
vs. JO15 + JO31, Figures 2B,C; Table 1). This finding suggests that scolopidia that have one vibration-sensitive and one static deflection-sensitive JO neuron as a pair (rather than a pair of vibration sensitive neurons or a pair of static deflectionsensitive neurons) are dominant in the JO.

\section{Organization of Single Subgroups of Vibration-Sensitive Johnston's Organ Neurons}

We next observed the organization of single subgroups of vibration-sensitive JO neurons using group-2 GAL4 strains (Figure 3; Table 1). When we used $R 74 C 10$, which labels most JO-A neurons (Ishikawa et al., 2017), 84.3\% of the selected scolopidia housed one labeled JO neuron and $14.8 \%$ had two. JO22, JO24, and JO26, which label only a small subset of JO-A (Kamikouchi et al., 2006), labeled only one JO neuron in all the selected scolopidia. This finding suggests that most, but not all, JO-A neurons are not paired with another JO-A neuron in the same scolopidium. When we used JO2, which labels most JO-B neurons (Kamikouchi et al., 2006), all the selected scolopidia housed one labeled JO neuron. All JO-B neurons are thus likely to be paired with a JO neuron of another subgroup.

To evaluate whether JO-A and JO-B are housed together in a single scolopidium, we combined two group-2 strains. When we combined $\mathrm{R} 74 \mathrm{C} 10$ and $\mathrm{JO} 2$, which label most JO-A and JO-B neurons, respectively, the ratio of single/double/ triple-labeled-neuron populations was not significantly altered
(Figure 3D; Table 1, $p=0.066$ for $R 74 C 10$ vs. $R 74 C 10+J O 2$, $p=0.082$ for $J O 2$ vs. $R 74 C 10+J O 2)$. This observation suggests that most scolopidia have either JO-A or JO-B as a vibrationsensitive JO neuron.

\section{DISCUSSION}

In the present study, we explored the organization of JO neurons in each scolopidium and revealed that most scolopidia had one vibration-sensitive JO neuron and one static deflection-sensitive JO neuron as a pair. This finding suggests that JO neurons are housed in a heterogenous manner that one JO neuron for hearing and another for sensing gravity and wind are paired within a single scolopidium. JO-B neurons, which selectively respond to low-frequency vibrations, were also singly housed in most scolopidia, suggesting a general rule that multiple neurons with similar response properties are not located in a single scolopidium. A deviation from this rule was observed for JO-A neurons; although most JO-A neurons were housed singly in each scolopidium, some JO-A neurons were paired with another JO-A neuron. A previous report revealed the heterogeneity of JO-A neurons at the anatomic and physiologic levels (Ishikawa et al., 2017), raising the possibility that two heterogeneous JO-A neurons could be housed together in a single scolopidium. Since our analysis was restricted to the scolopedia that are observable from particular orientations, we could not exclude the possibility that these rules might
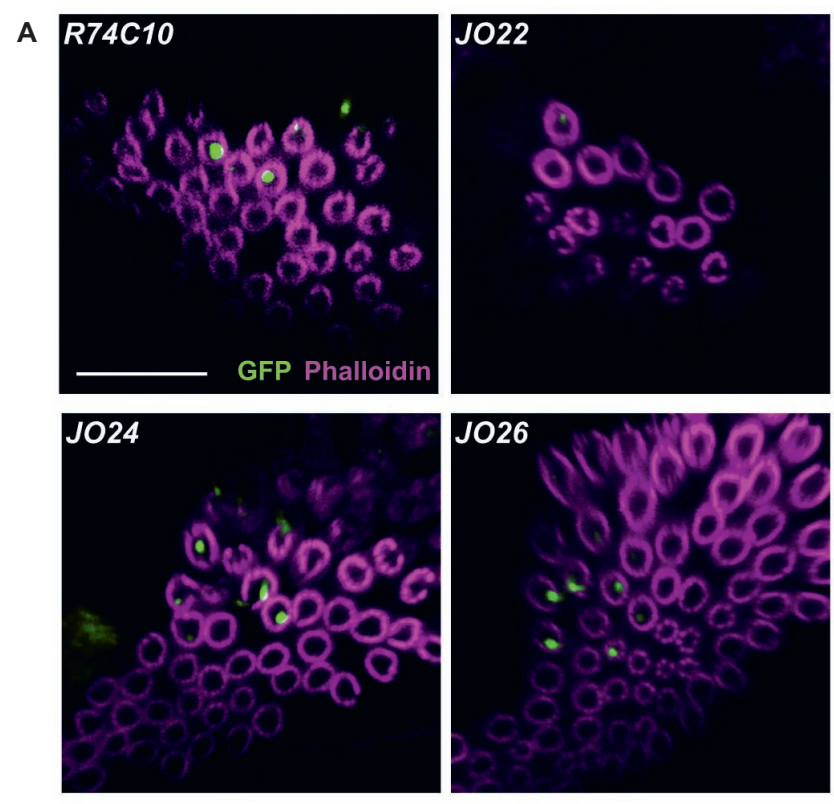
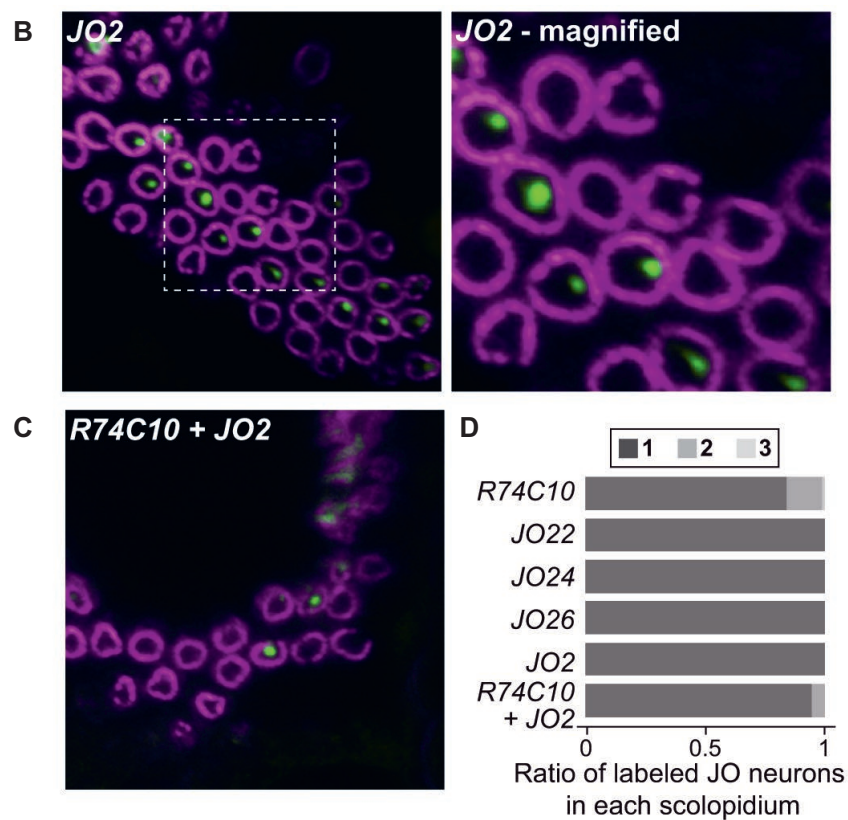

FIGURE 3 | Organization of different subgroups of vibration-sensitive JO neurons in scolopidia. (A-C) Labeled JO neurons in group-2 GAL4 driver strains that label vibration-sensitive JO-A (A), JO-B (B), and their combinations (C). Magnified view of JO2 is shown. Scale bar = $10 \mu \mathrm{m}$. (D) The ratio of one, two, and three labeled JO neurons per scolopidium in each GAL4 driver strain and a combination of two GAL4 driver strains. 
be true only in a specific JO neuron population. Further analyses are required to evaluate this possibility.

Mutual interference may occur between two sensory neurons housed in the same compartment (i.e., scolopidium), as the cilia of the two JO neurons in a scolopidium are in close contact and can influence each other through passive electrical interactions during stimulation. A previous study using an electrical model of a multi-neuron sensillum indicated that gathering identical neuron types in the same sensillum is generally disadvantageous, but may be advantageous if their thresholds differ (Vermeulen and Rospars, 2004). Indeed, insect olfactory sensilla house heterogeneous ORNs (de Bruyne et al., 2001). In Bombyx mori, the trichoid sensilla on the antennae house two ORNs, each sensitive to one of the two components of the sexual pheromone, e.g., bombykol or bombykal (Kaissling et al., 1978). In $D$. melanogaster, the ORNs in the olfactory sensilla are clustered in stereotyped combinations and activation of an ORN inhibits its neighboring ORN housed in the same sensillum (de Bruyne et al., 2001; Su et al., 2012). Further studies are needed to evaluate whether such passive (non-synaptic) electrical interactions occur between two JO neurons in a scolopidium.

\section{DATA AVAILABILITY STATEMENT}

The raw data supporting the conclusions of this article will be made available by the corresponding author upon reasonable request.

\section{REFERENCES}

Boekhoff-Falk, G., and Eberl, D. F. (2014). The Drosophila auditory system. Wiley Interdiscip. Rev. Dev. Biol. 3, 179-191. doi: 10.1002/wdev.128

Cheng, L. E., Song, W., Looger, L. L., Jan, L. Y., and Jan, Y. N. (2010). The role of the TRP channel NompC in Drosophila larval and adult locomotion. Neuron 67, 373-380. doi: 10.1016/j.neuron.2010.07.004

Coen, P., and Murthy, M. (2016). Singing on the fly: sensorimotor integration and acoustic communication in Drosophila. Curr. Opin. Neurobiol. 38, 38-45. doi: 10.1016/j.conb.2016.01.013

de Bruyne, M., Foster, K., and Carlson, J. R. (2001). Odor coding in the Drosophila antenna. Neuron 30, 537-552. doi: 10.1016/S0896-6273(01) 00289-6

Freeman, E. G., and Dahanukar, A. (2015). Molecular neurobiology of Drosophila taste. Curr. Opin. Neurobiol. 34, 140-148. doi: 10.1016/j. conb.2015.06.001

Gopfert, M. C., and Hennig, R. M. (2016). Hearing in insects. Annu. Rev. Entomol. 61, 257-276. doi: 10.1146/annurev-ento-010715-023631

Ishikawa, Y., and Kamikouchi, A. (2016). Auditory system of fruit flies. Hear. Res. 338, 1-8. doi: 10.1016/j.heares.2015.10.017

Ishikawa, Y., Okamoto, N., Nakamura, M., Kim, H., and Kamikouchi, A. (2017). Anatomic and physiological heterogeneity of subgroup - a auditory sensory neurons in fruit flies. Front. Neural Circuits 11:46. doi: 10.3389/ fncir.2017.00046

Kaissling, K. E., Kasang, G., Bestmann, H. J., Stransky, W., and Vostrowsky, O. (1978). A new pheromone of the silkworm moth Bombyx mori. Sci. Nat. 65, 382-384. doi: 10.1007/BF00439702

Kamikouchi, A., Inagaki, H. K., Effertz, T., Hendrich, O., Fiala, A., Gopfert, M. C., et al. (2009). The neural basis of Drosophila gravity-sensing and hearing. Nature 458, 165-171. doi: 10.1038/nature07810

\section{AUTHOR CONTRIBUTIONS}

$\mathrm{AK}$ and YI contributed to the conception and design of the study and wrote the manuscript. MF, JW, and AU performed the experiments. YI performed the statistical analysis. All authors contributed to manuscript revision, read and approved the submitted version.

\section{FUNDING}

This work was supported by MEXT KAKENHI Grants-in-Aid for Scientific Research (B) (Grant 16H04655 to AK and 18H02488 to YI), Scientific Research on Innovative Areas "Evolinguistics" (Grant 18H05069 to AK), "Systems Science of Bio-navigation" (Grant 19H04933 to AK), "Constrained and Directional Evolution" (Grant 18H04819 to YI), Challenging Research (Exploratory) (Grant 17K19450 to AK, and 17K19425 and 19K22453 to YI), Narishige Zoological Science Award to YI, and grant from the Naito Foundation and Takeda Science Foundation to AK.

\section{ACKNOWLEDGMENTS}

We thank Dr. Y. N. Jan and Bloomington Drosophila Stock Center for fly stocks, the Developmental Studies Hybridoma Bank for reagents, and $\mathrm{M}$. Kuno and Y. Maki for fly maintenance. We also thank Dr. M. P. Su, Dr. S. Sugiyama, and Dr. E. Matsuo for discussions.

Kamikouchi, A., Shimada, T., and Ito, K. (2006). Comprehensive classification of the auditory sensory projections in the brain of the fruit fly Drosophila melanogaster. J. Comp. Neurol. 499, 317-356. doi: 10.1002/cne.21075

Lu, T., Qiu, Y. T., Wang, G., Kwon, J. Y., Rutzler, M., Kwon, H. W., et al. (2007). Odor coding in the maxillary palp of the malaria vector mosquito Anopheles gambiae. Curr. Biol. 17, 1533-1544. doi: 10.1016/ j.cub.2007.07.062

Mamiya, A., and Dickinson, M. H. (2015). Antennal mechanosensory neurons mediate wing motor reflexes in flying Drosophila. J. Neurosci. 35, 7977-7991. doi: 10.1523/JNEUROSCI.0034-15.2015

Matsuo, E., Seki, H., Asai, T., Morimoto, T., Miyakawa, H., Ito, K., et al. (2016). Organization of projection neurons and local neurons of the primary auditory center in the fruit fly Drosophila melanogaster. J. Comp. Neurol. 524, 1099-1164. doi: 10.1002/cne.23955

Matsuo, E., Yamada, D., Ishikawa, Y., Asai, T., Ishimoto, H., and Kamikouchi, A. (2014). Identification of novel vibration- and deflection-sensitive neuronal subgroups in Johnston's organ of the fruit fly. Front. Physiol. 5:179. doi: 10.3389/fphys.2014.00179

Nikonov, A. A., and Leal, W. S. (2002). Peripheral coding of sex pheromone and a behavioral antagonist in the Japanese beetle, Popillia japonica. J. Chem. Ecol. 28, 1075-1089. doi: 10.1023/a:1015274104626

Patella, P., and Wilson, R. I. (2018). Functional maps of mechanosensory features in the Drosophila brain. Curr. Biol. 28, 1189-1203.e5. doi: 10.1016/j. cub.2018.02.074

Su, C. Y., Menuz, K., Reisert, J., and Carlson, J. R. (2012). Non-synaptic inhibition between grouped neurons in an olfactory circuit. Nature 492, 66-71. doi: 10.1038/nature11712

Todi, S. V., Sharma, Y., and Eberl, D. F. (2004). Anatomical and molecular design of the Drosophila antenna as a flagellar auditory organ. Microsc. Res. Tech. 63, 388-399. doi: 10.1002/jemt.20053 
Uga, S., and Kuwabara, M. (1965). On the fine structure of the chordotonal sensillum in antenna of Drosophila melanogaster. J. Electron Microsc. 14, 173-181.

Vermeulen, A., and Rospars, J. P. (2004). Why are insect olfactory receptor neurons grouped into sensilla? The teachings of a model investigating the effects of the electrical interaction between neurons on the transepithelial potential and the neuronal transmembrane potential. Eur. Biophys. J. 33, 633-643. doi: 10.1007/s00249-004-0405-4

Yorozu, S., Wong, A., Fischer, B. J., Dankert, H., Kernan, M. J., Kamikouchi, A., et al. (2009). Distinct sensory representations of wind and near-field sound in the Drosophila brain. Nature 458, 201-205. doi: 10.1038/nature07843
Conflict of Interest: The authors declare that the research was conducted in the absence of any commercial or financial relationships that could be construed as a potential conflict of interest.

Copyright (c) 2020 Ishikawa, Fujiwara, Wong, Ura and Kamikouchi. This is an openaccess article distributed under the terms of the Creative Commons Attribution License (CC BY). The use, distribution or reproduction in other forums is permitted, provided the original author(s) and the copyright owner(s) are credited and that the original publication in this journal is cited, in accordance with accepted academic practice. No use, distribution or reproduction is permitted which does not comply with these terms. 